\title{
The NOTCH3 Downstream Target HEYL Regulates Human Airway Epithelial Club Cell
}

\section{Differentiation}

Manish Bodas ${ }^{1}$, Bharathiraja Subramaniyan ${ }^{1}$, Andrew R. Moore ${ }^{1}$, Jordan P. Metcalf ${ }^{1,2}$, Sarah R. Ocañas $^{3}$, Willard M. Freeman ${ }^{2,4}$, Constantin Georgescu ${ }^{4}$, Jonathan D. Wren ${ }^{4}$, and Matthew S. Walters $^{1 \#}$

${ }^{1}$ Department of Medicine Section of Pulmonary, Critical Care \& Sleep Medicine University of Oklahoma Health Sciences Center Oklahoma City, OK, USA

${ }^{2}$ Oklahoma City Veterans Affairs Medical Center, Oklahoma City, OK, USA

${ }^{3}$ Department of Physiology

University of Oklahoma Health Sciences Center

Oklahoma City, OK, USA

${ }^{4}$ Genes \& Human Disease Research Program

Oklahoma Medical Research Foundation

Oklahoma City, OK, USA

\section{\#Correspondence:}

Dr. Matthew S Walters

Assistant Professor

800 N. Research Parkway,

Building 800, 4th Floor, Rm 410

Oklahoma City, OK 73104

Office: 405-271-3803 (on campus 13803)

Email: Matthew-S-Walters@ouhsc.edu

Keywords: NOTCH3 signaling, HEYL, Differentiation, Airway epithelium, Basal stem/progenitor cells, Club cells 


\begin{abstract}
Basal cells $(\mathrm{BC})$ are the resident stem/progenitor cells of the adult pseudostratified airway epithelium, whose differentiation program is orchestrated by the NOTCH signaling pathway. NOTCH3 receptor mediated signaling regulates $\mathrm{BC}$ to club cell differentiation; however, the downstream responses that regulate this process are largely unknown. In the present study we used an in vitro air-liquid interface model of the human pseudostratified airway epithelium to identify the NOTCH3-dependent downstream genes/pathways that regulate human BC to club cell differentiation. Activation of NOTCH3 signaling in BC via lentivirus-mediated over-expression of the active NOTCH3 intracellular domain (NICD3) promoted club cell differentiation. Bulk RNA-seq analysis of control vs NICD3 -transduced cells, identified 692 NICD3 responsive genes enriched for pathways linked to airway epithelial biology and differentiation including Wnt/ $\beta$ catenin Signaling. Expression of the classical NOTCH target HEYL increased in response to NOTCH3 activation and positively correlated with expression of the club cell marker SCGB1A1. Further, using single-cell RNA-seq, we report that $\mathrm{HEYL}^{+}$cells primarily clustered with $\mathrm{SCGB}_{1 \mathrm{~A}} 1^{+}$and $\mathrm{NOTCH}^{+}$cells. Moreover, HEYL protein co-localized with SCGB1A1 in ALI cultures in vitro and in the human and mouse airway epithelium in vivo. siRNA-mediated knockdown of HEYL in BC led to changes in epithelial structure including altered morphology and significant reductions in transepithelial electrical resistance and expression of tight junction related genes. Finally, HEYL knockdown significantly reduced the number of SCGB1A1+ club cells, along with a corresponding increase in $\mathrm{KRT}^{+} \mathrm{BC}$-intermediate cells. Overall, our data identifies NOTCH3-HEYL signaling as a key regulator of BC to club cell differentiation.
\end{abstract}




\section{Introduction}

The pseudostratified epithelium is a multi-cellular tissue that lines the conducting airways and functions as a barrier to protect the lung from environmental insults [1-4]. Basal cells (BC) are the resident stem/progenitor cells of the pseudostratified airway epithelium in humans and mice that initiate repair of the epithelium during homeostasis and following injury [1-9]. In response to specific stimuli, BC first differentiate into secretory club cells via BC-derived intermediate cells (also termed supra-BC or secretory primed-BC), which in turn undergo differentiation into either goblet secretory or ciliated cell lineages [5-9]. A reduction in the number of club cells is associated with human chronic lung disease, including asthma, chronic obstructive pulmonary disease (COPD) and idiopathic pulmonary fibrosis (IPF) [10-13]. Therefore, identifying the mechanisms that regulate $\mathrm{BC}$ to club cell differentiation are central to understanding the pathophysiology of chronic lung disease and the lung's response to injury.

The NOTCH signaling pathway regulates BC stem/progenitor function and cell fate decisions in the human and murine airway epithelium [5, 14-44]. Canonical NOTCH signaling is initiated by the binding of a ligand (DLL1, DLL3, DLL4, JAG1 or JAG2) to one of the four receptors (NOTCH1-4) located on the surface of a neighboring cell. This leads to proteolytic cleavage of the receptor and release of the Notch intracellular domain (NICD) into the cytoplasm $[45,46]$. The NICD then translocates to the nucleus and regulates expression of multiple downstream genes [45, 46]. Despite the knowledge that activation of NOTCH3 signaling is critical for BC to club cell differentiation $[5,19,27]$, the downstream genes and pathways that regulate this process are unknown. The present study was designed to address this gap in our knowledge. Using the in vitro air-liquid interface (ALI) system to mimic the human pseudostratified airway epithelium, we have characterized the NOTCH3-dependent downstream genes/pathways in differentiating BC and 
identified the transcription factor HEYL as a NOTCH3 target that regulates club cell differentiation.

\section{Materials and Methods}

\section{Primary human bronchial epithelial cell (HBEC) culture}

Primary HBECs from normal, nonsmokers were purchased commercially (catalog number CC2540, Lonza, Morristown, NJ, USA). The cells were maintained in BronchiaLife ${ }^{\mathrm{TM}}$ epithelial airway medium (BLEAM) (catalog number LL-0023, Lifeline ${ }^{\circledR}$ Cell Technology, Oceanside, CA, USA) supplemented with Penicillin (100 I.U./ml) - Streptomycin (100 $\mu \mathrm{g} / \mathrm{ml})$ under standard cell culture conditions as previously described [14]. All experiments were performed with either Passage 2 or 3 cells. In total, $n=9$ cell donors were used in this study with the following lot numbers and demographics: Donor 1 (lot number: 0000501936, female, Hispanic, 42 years old), Donor 2 (lot number: 0000544414, male, Caucasian, 48 years old), Donor 3 (lot number: 0000619261, male, Caucasian, 53 years old), Donor 4 (lot number: 0000543643, female, Caucasian, 57 years old), Donor 5 (lot number: 0000613375, female, Black, 65 years old), Donor 6 (lot number: 0000529235, female, Black, 67 years old), Donor 7 (lot number: 0000608196, male, Caucasian, 67 years old), Donor 8 (lot number: 0000420927, female, Hispanic, 69 years old) and Donor 9 (lot number: 0000444771, male, Black, 69 years old).

\section{Air-liquid interface (ALI) culture}

HBECs were differentiated using ALI culture to generate a pseudostratified airway epithelium as described previously [14]. Briefly, $1 \times 10^{5}$ cells in $100 \mu$ of BLEAM media were seeded in the apical chamber of a $0.4 \mu \mathrm{m}$ pore-sized Transwell ${ }^{\circledR}$ insert (catalog number 3470 , Corning ${ }^{\circledR}$, Corning, NY, USA) pre-coated with human type IV collagen (catalog number C7521, Sigma Aldrich, St. Louis, MO, USA) with $1 \mathrm{ml}$ of BLEAM in the basolateral chamber (ALI day -2). The 
next day, fresh BLEAM media was replaced in the apical and basolateral chambers $(100 \mu 1$ and 1 $\mathrm{ml}$, respectively). After two days of submerged culture, media from the apical chamber was removed to expose the cells to air (ALI day 0), and $1 \mathrm{ml}$ of Human Bronchial/Tracheal Epithelial Cell (HBTEC) ALI differentiation medium (Cat\# LM-0050, Lifeline ${ }^{\circledR}$ Cell Technology) supplemented with Penicillin (100 I.U./ml) - Streptomycin (100 $\mu \mathrm{g} / \mathrm{ml})$ was added to the basolateral chamber. The media was replaced every other day and the cells allowed to differentiate for up to 9 days.

\section{RNA extraction, cDNA synthesis and quantitative PCR analysis}

Total RNA was extracted via direct lysis of cells in the ALI well using the PureLink ${ }^{\mathrm{TM}}$ RNA mini kit (catalog number 12183018A, Thermo Fisher Scientific, Waltham, MA, USA) and included DNase treatment (catalog number 12185-010, Thermo Fisher Scientific) on the column to remove contaminating genomic DNA. Complementary DNA (cDNA) was generated from 100 ng of total RNA per sample using random hexamers (Applied Biosystems ${ }^{\mathrm{TM}}$ High Capacity cDNA Reverse Transcription Kit, catalog number 4374966, Thermo Fisher Scientific) and subsequent quantitative PCR (qPCR) analysis performed using iTaq $^{\mathrm{TM}}$ Universal SYBR $^{\circledR}$ Green supermix (catalog number 1725124, Bio-Rad, Hercules, CA, USA) on the Bio-Rad CFX96 Touch $^{\text {TM }}$ RealTime PCR system. All samples were analyzed in duplicate with relative expression levels determined using the $\mathrm{dCt}$ method with Actin Beta (ACTB) as the endogenous control. The following PrimePCR ${ }^{\mathrm{TM}}$ gene-specific primers were purchased from Bio-Rad: ACTB (qHsaCED0036269), SCGB1A1 (qHsaCID0018013), NOTCH3 (qHsaCID0006529), HEY1 (qHsaCED0046240), HEYL (qHsaCID0006092), NRARP (qHsaCED0048313), KRT5 (qHsaCED0047798), CLDN3 (qHsaCEP0032466), OCLN $\quad$ (qHsaCEP0041012), $\quad$ TJP1 (qHsaCIP0031627), TJP2 (qHsaCIP0026125), $\quad$ TJP3 (qHsaCIP0030881), PARD3 
(qHsaCIP0039350) and PARD6B (qHsaCEP0051277), and the assays performed using the manufacturer's recommend cycling parameters.

\section{Lentivirus-based overexpression of NICD3}

Generation of Control (empty vector) or NICD3 expressing replication deficient lentiviruses was described previously [14]. Stocks of Lenti-Control and Lenti-NICD3 were titrated by qPCR (catalog number LV900, ABM ${ }^{\circledR}$ good, Richmond, BC, Canada) and their infectivity confirmed by flow cytometry via quantification of $\mathrm{GFP}^{+}$cells. Cells were infected at a multiplicity of infection of $50\left(5 \times 10^{6}\right.$ viral genomes $/ 1 \times 10^{5}$ cells $)$ at the time of seeding the cells on ALI to ensure that $>90 \%$ of cells were infected $\left(\mathrm{GFP}^{+}\right)$with each virus. To aid virus infection, the BLEAM media was supplemented with $2 \mu \mathrm{g} / \mathrm{ml}$ of polybrene (catalog number TR-1003-G, Sigma Aldrich). The next day, fresh media was added to the wells and the standard ALI protocol was continued until the day of harvest.

\section{Bulk RNA sequencing (Bulk RNA-seq)}

Control or NICD3-transduced cells ( $n=6$ donors) were harvested as a function of time on ALI (day $0,3,5$ and 7) and the genome-wide transcriptome changes in response to NICD3 expression assessed by bulk RNA-seq. Total RNA was extracted from each sample (described above) and bulk RNA-seq was performed on a NextSeq 500 Flowcell, High SR75 (Illumina, San Diego, CA, USA) following library preparation using the QuantSeq 3' mRNA-Seq Library Prep Kit FWD for Illumina (Lexogen, Vienna, Austria). RNA-seq data processing followed the guidelines and practices of the ENCODE and modENCODE consortia regarding proper experimental replication, sequencing depth, data and metadata reporting, and data quality assessment (https://www.encodeproject.org/documents/cede0cbe-d324-4ce7-ace4-f0c3eddf5972/). ～Raw sequencing reads (in a FASTQ format) were trimmed of residual adaptor sequences using Scythe 
software. Low quality bases at the beginning or the end of sequencing reads were removed using sickle then the quality of remaining reads was confirmed with FastQC. Further processing of quality sequencing reads was performed with utilities provided by the Tuxedo Suite software. Reads were aligned to the Homo sapiens genome reference (GRCh38/hg38) using the TopHat component, then cuffquant and cuffdiff were utilized for gene-level read counting and differentially expression analysis. Genes that were significantly differentially expressed in response to NICD3 for at least one time point were determined using a threshold on the false discovery rate (FDR) adjusted p-value of 0.05 (FDR adjusted $\mathrm{p}<0.05)$. Ingenuity Pathway Analysis (IPA) (Qiagen, Redwood City, CA, USA) was used to identify molecular pathways altered in response to NICD3 using an unrestricted analysis.

\section{Single cell RNA sequencing (scRNA-seq)}

HBECs from a single donor were cultured on ALI for 9 days and harvested for scRNA-seq analysis. To generate single cell suspensions, the cells were trypsinized for 3 mins and following neutralization passed through a Flowmi ${ }^{\mathrm{TM}}$ tip strainer $(40 \mu \mathrm{m}$ porosity, catalog number H136800040, SP Bel-Art, Wayne, NJ, USA). Cells were counted on a hemocytometer prior to diluting cells to 800 cells/ $\mu 1$ in $0.1 \%$ BSA/PBS buffer for scRNA-seq library preparation with Chromium Single Cell 3' Reagent Kits v3 (catalog number PN-1000075, 10X Genomics, Pleasanton, CA, USA). scRNA-seq libraries were generated according to 10X Genomics User Guide CG000183

Rev C. Briefly, a reaction mix containing 12,800 cells was loaded into a Chromium Chip B, targeting cell recovery of 8000 cells. After generating Gel Beads-in-emulsion (GEMS) on the Chromium controller, GEMS were transferred to PCR tubes (catalog number 951010022, Eppendorf, Hamburg, Germany) for GEM-Reverse Transcription (GEM-RT). After cleanup with Dynabeads MyOne Silane (catalog number PN-2000048, 10X Genomics), cDNA was amplified 
with 11 cycles. Amplified cDNA was cleaned with 0.6X SPRISelect reagent (catalog number B23318, Beckman Coulter, Pasadena, CA, USA). Cleaned cDNA was then quality checked on an Agilent Tapestation 4150 (catalog number G2992AA, Agilent, Santa Clara, CA, USA) using a High Sensitivity D5000 ScreenTape (catalog number 5067-5592, Agilent) and quantified using Qubit dsDNA High Sensitivity Assay Kit (catalog number Q32851, Thermo Fisher Scientific) read on a Qubit 4 Fluorometer (catalog number Q33238, Thermo Fisher Scientific). An aliquot of 25\% of the cleaned cDNA was used for library construction (fragmentation, end repair, A-tailing, adaptor ligation), according to the manufacturer's instructions. Libraries were indexed (catalog number PN-220103, 10X Genomics) using 10 cycles of PCR. Amplified libraries were cleaned with SPRISelect reagent using a double-sided size selection protocol. Cleaned libraries were quality checked on an Agilent Tapestation 4150 using a High Sensitivity D1000 ScreenTape (catalog number 5067-5584, Agilent) and quantified using Qubit dsDNA High Sensitivity Assay Kit. Libraries were diluted to 4nM prior to sequencing on a NovaSeq 6000 S1 flow cell with 28 cycles for read 1 and 90 cycles for read 2. Cellranger v3.1.0 (10X Genomics) cellranger mkfastq was used to demultiplex fastq files from raw base call (BCL) files. Fastq files were then aligned to Homo sapiens (human) genome assembly GRCh38-3.0.0 (hg38), filtered, and barcodes/UMIs counted using the cellranger count function. Summary metrics revealed 11,567 cells with an average of 8452 reads/cell and a median of 1382 genes/cell. scRNA-seq data was visualized using Loupe Cell Browser (10X Genomics).

\section{Data availability}

The raw data from the bulk RNA-seq and scRNA-seq studies are publicly available at the Gene Expression Omnibus (GEO) site (http://www.ncbi.nlm.nih.gov/geo/), accession number GSE168128. 


\section{siRNA mediated knockdown of NOTCH3 and HEYL}

HBECs were transfected with either 1 pmol of Silencer ${ }^{\circledR}$ Select Negative Control No.1 siRNA (catalog number 4390844), NOTCH3 siRNA (catalog number 4392420; assay ID s453556), or HEYL siRNA (catalog number 4392420; assay ID s223702) using Lipofectamine RNAiMax Reagent (catalog number 13778075) and OptiMEM media (catalog number 31985070) (all from Thermo Fisher Scientific) at the time of seeding the cells in ALI culture. The following day, media in both apical and basolateral chambers was replaced with fresh BLEAM media, and the standard ALI protocol was continued until day of harvest (ALI day 7).

\section{Immunofluorescence staining and quantification of differentiation}

Immunofluorescent staining of either differentiating cells in ALI wells or of the paraffin embedded human bronchus from healthy nonsmokers (Donor 1: Age 27, Female; Donor 2: Age 40, Female and Donor 3: Age 27, Female; catalog number HuFPT111, US Biomax, Inc., Rockville, MD, USA), or mouse trachea (C57BL/6, Normal, Female, 12 weeks) sections was performed as described [14]. For mouse trachea, the sections were subjected to an additional 1 hour of blocking at room temperature, with $20 \mu \mathrm{g} / \mathrm{ml}$ Affinipure Fab fragment goat anti-mouse $\operatorname{IgG}(\mathrm{H}+\mathrm{L})(\mathrm{catalog}$ number 115-007-003, Jackson ImmunoResearch Laboratories, Inc. West Grove, PA, USA). The following primary antibodies were used: HEYL $(5 \mu \mathrm{g} / \mathrm{ml}$, catalog number H00026508-M03, Abnova, Taipei, Taiwan), SCGB1A1 (5 $\mu \mathrm{g} / \mathrm{ml}$, catalog number RD181022220-01, BioVendor LLC, Asheville, NC, USA), KRT5 (2 $\mu \mathrm{g} / \mathrm{ml}$, catalog number PA1-37974, Thermo Fisher Scientific) and KRT8 (5 $\mu \mathrm{g} / \mathrm{ml}$, catalog number NBP2-16094, Novus Biologicals, Centennial, CO, USA). Isotype matched mouse (catalog number 401402, BioLegend) or rabbit IgG (catalog number 02-6102, Invitrogen) were used as a negative control. To visualize antibody binding the following secondary antibodies were used: goat anti-mouse Alexa Fluor $488(2 \mu \mathrm{g} / \mathrm{ml}$, catalog 
number A11029, Thermo Fisher Scientific) and goat anti-rabbit Alexa Fluor $546(2 \mu \mathrm{g} / \mathrm{ml}$ catalog number A11035, Thermo Fisher Scientific). The cell nuclei were counterstained with DAPI (1 $\mu \mathrm{g} / \mathrm{ml}$, catalog number 62248, Thermo Fisher Scientific). Images were taken using an Olympus BX43 upright fluorescent microscope (Olympus Corporation). The number of cells positive for each marker of interest was counted using the ImageJ software (version 1.8.0_112, NIH), and normalized to the number of nuclei as described [14].

\section{Transepithelial electrical resistance (TEER)}

TEER was measured using the ENDOHM-6G and EVOM2 apparatus (World Precision Instruments, Sarasota, FL, USA) according to the manufacturer's guidelines. The resistance (ohms) of an empty Transwell ${ }^{\circledR}$ insert (with no cells) was subtracted from each sample to calculate the true tissue resistance.

\section{Statistics}

Statistical analysis was performed by two-tailed Mann Whitney U test using the GraphPad Prism version 8.0. A p value of $\leq 0.05$ was considered significant.

\section{Results}

To identify downstream genes and pathways that regulate NOTCH3-dependent differentiation of BC into club cells, bulk RNA-seq was performed on HBECs infected with control lentivirus or lentivirus expressing the constitutively active NICD3 as a function of time on ALI culture (day 0, 3, 5 and 7) (Fig. 1A-B). Quantitative PCR analysis of the club cell marker SCGB1A1 demonstrated increased club cell differentiation as a consequence of NICD3 over-expression in each HBEC donor (Fig. 1C). Comparison of NICD3-overexpressing vs. control cells identified 2319 genes with a significant (FDR adjusted $\mathrm{p}<0.05)$ expression change for at least one time point. For discovery 
purposes we increased the statistical stringency (FDR adjusted $\mathrm{p}<0.005)$ which reduced our list to 692 differentially expressed genes in response to NICD3 expression (Supplemental File 1). Analysis of the 692 gene set identified enrichment of molecular pathways including, "Hepatic Fibrosis / Hepatic Stellate Cell Activation”, "Inhibition of Matrix Metalloproteases”, "NOTCH Signaling", "Integrin Signaling" and "Wnt/ $\beta$-catenin Signaling” (Fig. 1D and Supplemental File 2). Expression of genes from each of these pathways correlated either positively or negatively with expression of SCGB1A1 in response to NICD3 expression (Fig. 1E). Further confirming the specificity of these downstream responses, siRNA knockdown of NOTCH3 (>70\% knockdown efficiency in each experiment) led to an opposite effect to NICD3 over-expression, and decreased the expression of SCGB1A1 (0.38 fold) and the classical NOTCH downstream targets HEY1 (0.51 fold), HEYL (0.39 fold) and NRARP (0.40 fold) (Fig. 1F).

HEYL expression is upregulated in the murine tracheal airway epithelium following injury $[32,34]$. However, the function of HEYL in the context of human lung differentiation is unknown. Quantitative PCR analysis showed that HEYL expression increased as a function of time in tandem with SCGB1A1 and NOTCH3 during HBEC differentiation on ALI (Fig. 2A). Furthermore, despite the low read depth limiting the detection of HEYL expression, scRNA-seq analysis of ALI day 9 cultures demonstrated that $\mathrm{HEYL}^{+}$cells predominantly grouped with cells positive for SCGB1A1 and NOTCH3 in clusters 1 and 2 (Fig. 2B-F). Finally, co-immunofluorescent staining of in vitro ALI cultures (Fig. 2G) and in vivo human bronchial (Fig. 3A) and mouse tracheal epithelium (Fig. 3B) demonstrated that HEYL localizes with SCGB1A1 ${ }^{+}$club cells. Combined, these data suggest that induction of HEYL expression may regulate differentiation of BC into club cells. To test this hypothesis, HBECs were transfected with either control siRNA or HEYL specific siRNA and then cultured for 7 days on ALI. Compared to siRNA Control transfected cells, HEYL 
expression was significantly suppressed (0.06 fold) in siRNA HEYL transfected cells (Fig. 4A). Knockdown of HEYL led to changes in epithelial structure including altered morphology (Fig. 4B) and significant reductions in TEER (Fig. 4C) and expression of tight junction related genes (Fig. 4D). Furthermore, knockdown of HEYL significantly impaired club cell differentiation with a reduction in club cell numbers ( 0.53 fold) (Fig. 4E). While HEYL knockdown had no significant effect on the number of $\mathrm{KRT}^{+} \mathrm{BC}(\mathrm{Fig} .4 \mathrm{~F})$, we observed an increase in the numbers of $\mathrm{KRT}^{+}$ BC-intermediate cells (2.42 fold) (Fig. 4G). Combined, these data suggest that HEYL is required for efficient differentiation of BC into club cells.

\section{Discussion}

NOTCH3 receptor signaling plays a key role in regulating differentiation of the pseudostratified airway epithelium during health and in chronic lung diseases, including asthma, COPD and IPF $[5,14,19,23,27,33]$. To date, studies have shown that NOTCH3 signaling impacts airway epithelial differentiation in a context dependent manner with both suppression and activation of NOTCH3 signaling leading to pathological changes in airway epithelial structure [5, $14,19,23,27,33]$. Therefore, greater understanding of the downstream genes and pathways that regulate NOTCH3-dependent differentiation are critical for understanding the mechanisms driving airway epithelial remodeling associated with chronic lung disease.

Based on the knowledge that NOTCH3 is an important regulator of club cell differentiation [5, 19, 27], we characterized the NOTCH3-dependent downstream genes/pathways in BC stem/progenitor cells as they differentiate into club cells on ALI culture. Bulk RNA-seq analysis identified 692 genes regulated in response to NOTCH3 activation that were enriched in molecular pathways associated with regulating airway epithelial structure and stem/progenitor cell function 
[24, 47-50]. These include "Hepatic Fibrosis/Hepatic Stellate Cell Activation”, "Inhibition of Matrix Metalloproteases" and "Integrin Signaling" [47, 48, 51]. Genes associated with these pathways play an important role in regulating the extracellular matrix (ECM), a structural scaffold that plays a critical role in regulating the growth, differentiation and function of airway epithelial cells $[52,53]$. Furthermore, alterations in ECM structure is associated with the pathophysiology of multiple chronic lung diseases including asthma, COPD and IPF [52, 54]. Vera et al [55] recently reported a pathogenic role for NOTCH3 signaling in fibroblast activation and pulmonary fibrosis. Using a bleomycin-induced model of pulmonary fibrosis, they report that Notch3-deficient mice were protected from bleomycin-induced pulmonary fibrosis and had reduced numbers of $\alpha$ SMApositive myofibroblasts compared to control fibrotic lungs. While mesenchymal cells (e.g., fibroblasts) are the major regulators of ECM deposition and organization, our data suggest that NOTCH3 signaling may have a similar role in regulating airway epithelial-derived ECM production. However, further studies are required to investigate the role of NOTCH3 in regulating these processes. Similar to NOTCH signaling, the Wnt/ $\beta$-catenin signaling pathway also plays a crucial role in regulating cell fate decisions in the human lung $[50,56,57]$. Therefore, our data demonstrating NOTCH3 activation modulates expression of multiple genes in the Wnt/ $\beta$-catenin signaling pathway suggests that NOTCH3 may cross-talk with this pathway to regulate airway epithelial differentiation.

At the single gene level, we also identified the transcription factor HEYL as a downstream target of NOTCH3 and a regulator of club cell differentiation. Expression of HEYL increases during differentiation on ALI culture in tandem with NOTCH3 and SCGB1A1 expression. Furthermore, HEYL is expressed in club cells in both the human (in vitro and in vivo) and mouse airway epithelium. Finally, knockdown of HEYL leads to a disruption in airway epithelial 
structure, as evident by decreased TEER values and reduced expression of tight junction genes, suggesting that HEYL directly regulates tight junction formation in the airway epithelium. Alternatively, the changes observed may be a consequence of decreased club cell differentiation and a corresponding increase in $\mathrm{KRT}^{+} \mathrm{BC}$-intermediate cell numbers. Human studies have shown that HEYL regulates differentiation of fetal neural stem cells [58] as well as proliferation of breast, prostate and liver cancer cells [59-61]. Further, expression of HEYL is regulated in a NOTCHindependent manner by TGF $\beta$ signaling [59, 60], BMP signaling [62], and epigenetically via LSD1-mediated histone methylation [58] and DNA methylation of the promoter region [60, 63]. Expression of Heyl is upregulated in the murine tracheal airway epithelium following polidocanol and sulphur dioxide induced injury $[32,34]$, suggesting that Heyl may function in the regeneration response of the airway epithelium. However, this hypothesis has not been directly tested. Mori et al [27] reported that NOTCH3 signaling in the murine airway epithelium was critical for priming of $\mathrm{BC}$ differentiation into club cells and that Notch3 knockout mice had increased numbers of $\mathrm{KRT}^{+}$undifferentiated progenitor cells in the airway epithelium compared to wild-type mice. These data support our findings whereby knockdown of HEYL leads to decreased club cell numbers and increased numbers of $\mathrm{KRT}^{+}$cells. Combined, these data suggest that NOTCH3/HEYL signaling is critical for regulating differentiation of BC into club cells and may be an early event in the regeneration response of the airway epithelium following injury. Therefore, future studies are required to explore the role of HEYL in regulating NOTCH3-dependent airway epithelial remodeling associated with human chronic lung disease. 
Acknowledgements: The authors thank Drs. Linda Thompson, Dean Dawson, Lorin Olson, and Xiao-Hong Sun at the Oklahoma Medical Research Foundation (OMRF) for discussions, guidance, and support. We also thank the Clinical Genomics Core and Imaging Core Facility at OMRF for assistance in sample processing related to our RNA-seq and histology studies.

\section{Declarations}

Funding: This work was supported by the following grants awarded to MSW: NIH/NIGMS COBRE (GM103636, Project 4), Oklahoma Center for Adult Stem Cell Research (OCASCR) Grant, College of Medicine Alumni Association (COMAA) Research Grant, Presbyterian Health Foundation (PHF) New Investigator Seed Grant and a Pilot Grant Award through the Genomic Sciences Core of the OK Nathan Shock Center (P30AG050911). The funders had no role in study design, data collection, data analysis, decision to publish, or preparation of the manuscript.

Conflicts of Interest/Competing interests: All the authors declare they have no conflict of interest in relation to the subject matter or materials discussed in this manuscript.

Ethics Approval: Not applicable.

Consent to Participate: Not applicable.

Consent for Publication: All the authors have provided consent for publication.

Availability of data and material: The raw data from the bulk RNA-seq and scRNA-seq studies are publicly available at the Gene Expression Omnibus (GEO) site (http://www.ncbi.nlm.nih.gov/geo/), accession number GSE168128.

Code availability: Not applicable. 
Author contributions: conception of study and experimental design: M.B. and M.S.W.; performed experiments: M.B., B.S., A.R.M., S.R.O., and M.S.W.; data analysis and/or interpretation: M.B., B.S., A.R.M., J.P.M., S.R.O., W.M.F., C.G., J.D.W. and M.S.W.; writing and preparation of original draft manuscript: M.B. and M.S.W. All authors have reviewed, critiqued, and approved the final manuscript. 


\section{Figure Legends}

Fig. 1. Activation of NOTCH3 signaling promotes club cell differentiation. (A) Experimental strategy. Primary HBECs (n=6 donors) were infected on air-liquid interface (ALI) with either control lentivirus (Lenti-Control) or lentivirus expressing the activated NOTCH3 NICD (LentiNICD3). The cells were then harvested as a function of time (ALI day 0, 3, 5 and 7) for subsequent analysis by qPCR and bulk RNA-seq. (B) GFP positivity of Lenti-Control and Lenti-NICD3 infected cells. Scale bar $=100 \mu \mathrm{m}$. (C) qPCR of SCGB1A1 (club cell marker) expression in LentiControl and Lenti-NICD3 infected cells from each HBEC donor. (D) Pathways enriched in the 692 NICD3-responsive gene list identified by bulk RNA-seq on the basis of Ingenuity Pathway Analysis (IPA). Shown are the top ten IPA-enriched pathways based on P value (log-transformed).

(E) Heatmap analysis of specific genes from the pathways highlighted in panel D. (F) HBECs ( $\mathrm{n}=3$ donors) were either transfected with control (siCon) or NOTCH3 (siNOTCH3) specific siRNA during seeding on ALI culture. At ALI day 7 the cells were harvested for qPCR analysis of NOTCH3, SCGB1A1, HEY1, HEYL and NRARP. For each donor, the data is presented as fold-change in expression compared to siCon cells. ${ }^{*} \mathrm{p}<0.05$.

Fig. 2. Expression of the NOTCH3 target HEYL correlates with club cell differentiation. (A) Primary HBECs ( $\mathrm{n}=4$ donors) were cultured on air-liquid interface (ALI) for 7 days. The cells were harvested at ALI day 0 and day 7 for qPCR analysis of KRT5 (basal cell marker), SCGB1A1 (club cell marker), NOTCH3 and HEYL. For each donor, the data is presented as fold-change in expression compared to ALI day 0. $* \mathrm{p}<0.05$. (B-F) scRNA-seq analysis of HBECs cultured on ALI for 9 days. (B) UMAP clustering using K-means 6 clustering approach of 11,567 cells from a single HBEC donor. (C) Expression of KRT5 (basal cell marker). (D) Expression of SCGB1A1 (club cell marker). (E) Expression of NOTCH3. (F) Expression of HEYL. (G) Immunofluorescent 
staining of SCGB1A1 (red), HEYL (green) and nuclei (blue, DAPI) in ALI day 7 cells. Four representative images (I-IV) are shown. Scale bar $=20 \mu \mathrm{m}$.

Fig. 3. HEYL co-localizes with SCGB1A1 in the human and mouse airway epithelium in vivo. Immunofluorescent staining of SCGB1A1 (red), HEYL (green) and nuclei (blue, DAPI) in (A) human bronchus sections ( $\mathrm{n}=3$; donors) and $(\mathbf{B})$ mouse trachea $(\mathrm{n}=3$ mice $)$. Scale bar $=20 \mu \mathrm{m}$.

Fig. 4. siRNA-mediated knockdown of HEYL suppresses club cell differentiation. Primary HBECs ( $n=4$ donors) were transfected with either control (siCon) or HEYL (siHEYL) specific siRNA during seeding on ALI culture. At ALI day 7 the cells harvested for analysis. (A) qPCR analysis of HEYL. For each donor, the data are presented as fold-change in expression compared to siCon cells. ${ }^{*} \mathrm{p}<0.05$. (B) Morphology of the cells following knockdown of HEYL expression. Scale bar $=100 \mu \mathrm{m}$. (C) Transepithelial electrical resistance (TEER). For each donor, the resistance (ohms) is plotted as percentage $(\%)$ resistance compared to siCon cells. $* \mathrm{p}<0.05$. (D) qPCR analysis of the tight junction related genes CLDN3, OCLN, TJP1, TJP2, TJP3, PARD3 and PARD6B. For each donor, the data are presented as fold-change in expression compared to siCon cells. * $\mathrm{p}<0.05$. (E-G) Immunofluorescent staining and quantification of club cells (SCGB1A1, red), basal cells (KRT5, red) and basal-intermediate cells (KRT8, red). Nuclei are stained blue with DAPI. For each donor, the data are presented as fold-change in cell numbers compared to siCon cells. Scale bar $=20 \mu \mathrm{m} . * \mathrm{p}<0.05$. 


\section{References}

1. Hogan, B.L., et al., Repair and regeneration of the respiratory system: complexity, plasticity, and mechanisms of lung stem cell function. Cell Stem Cell, 2014. 15(2): p. 12338.

2. Rock, J.R., S.H. Randell, and B.L. Hogan, Airway basal stem cells: a perspective on their roles in epithelial homeostasis and remodeling. Dis Model Mech, 2010. 3(9-10): p. 545-56.

3. Tata, P.R. and J. Rajagopal, Plasticity in the lung: making and breaking cell identity. Development, 2017. 144(5): p. 755-766.

4. Whitsett, J.A., et al., Building and Regenerating the Lung Cell by Cell. Physiol Rev, 2019. 99(1): p. 513-554.

5. Carraro, G., et al., Single-Cell Reconstruction of Human Basal Cell Diversity in Normal and Idiopathic Pulmonary Fibrosis Lungs. Am J Respir Crit Care Med, 2020. 202(11): p. 1540-1550.

6. Montoro, D.T., et al., A revised airway epithelial hierarchy includes CFTR-expressing ionocytes. Nature, 2018. 560(7718): p. 319-324.

7. Plasschaert, L.W., et al., A single-cell atlas of the airway epithelium reveals the CFTR-rich pulmonary ionocyte. Nature, 2018. 560(7718): p. 377-381.

8. Ruiz Garcia, S., et al., Novel dynamics of human mucociliary differentiation revealed by single-cell RNA sequencing of nasal epithelial cultures. Development, 2019. 146(20).

9. Zaragosi, L.E., M. Deprez, and P. Barbry, Using single-cell RNA sequencing to unravel cell lineage relationships in the respiratory tract. Biochem Soc Trans, 2020. 48(1): p. 327336.

10. Gamez, A.S., et al., Supplementing defect in club cell secretory protein attenuates airway inflammation in COPD. Chest, 2015. 147(6): p. 1467-1476.

11. Pilette, C., et al., Reduced epithelial expression of secretory component in small airways correlates with airflow obstruction in chronic obstructive pulmonary disease. Am J Respir Crit Care Med, 2001. 163(1): p. 185-94.

12. Reynaud, P., et al., Club Cell Loss as a Feature of Bronchiolization in ILD. Frontiers in Immunology, 2021. 12(311).

13. Shijubo, N., et al., Clara cell protein-positive epithelial cells are reduced in small airways of asthmatics. Am J Respir Crit Care Med, 1999. 160(3): p. 930-3.

14. Bodas, M., et al., Cigarette Smoke Activates NOTCH3 to Promote Goblet Cell Differentiation in Human Airway Epithelial Cells. Am J Respir Cell Mol Biol, 2021. 
15. Pardo-Saganta, A., et al., Injury induces direct lineage segregation of functionally distinct airway basal stem/progenitor cell subpopulations. Cell Stem Cell, 2015. 16(2): p. 184-97.

16. Boucherat, O., J. Chakir, and L. Jeannotte, The loss of Hoxa5 function promotes Notchdependent goblet cell metaplasia in lung airways. Biol Open, 2012. 1(7): p. 677-91.

17. Carrer, M., et al., Antisense Oligonucleotides Targeting Jagged 1 Reduce House Dust Miteinduced Goblet Cell Metaplasia in the Adult Murine Lung. Am J Respir Cell Mol Biol, 2020. 63(1): p. 46-56.

18. Danahay, H., et al., Notch2 is required for inflammatory cytokine-driven goblet cell metaplasia in the lung. Cell Rep, 2015. 10(2): p. 239-52.

19. Gomi, K., et al., Activation of NOTCH1 or NOTCH3 signaling skews human airway basal cell differentiation toward a secretory pathway. PLoS One, 2015. 10(2): p. e0116507.

20. Gomi, K., et al., JAG1-Mediated Notch Signaling Regulates Secretory Cell Differentiation of the Human Airway Epithelium. Stem Cell Rev Rep, 2016. 12(4): p. 454-63.

21. Guseh, J.S., et al., Notch signaling promotes airway mucous metaplasia and inhibits alveolar development. Development, 2009. 136(10): p. 1751-9.

22. Ito, T., et al., Basic helix-loop-helix transcription factors regulate the neuroendocrine differentiation of fetal mouse pulmonary epithelium. Development, 2000. 127(18): p. 391321.

23. Jing, Y., et al., NOTCH3 contributes to rhinovirus-induced goblet cell hyperplasia in COPD airway epithelial cells. Thorax, 2019. 74(1): p. 18-32.

24. Kiyokawa, H. and M. Morimoto, Notch signaling in the mammalian respiratory system, specifically the trachea and lungs, in development, homeostasis, regeneration, and disease. Dev Growth Differ, 2020. 62(1): p. 67-79.

25. Lafkas, D., et al., Therapeutic antibodies reveal Notch control of transdifferentiation in the adult lung. Nature, 2015. 528(7580): p. 127-31.

26. Marcet, B., et al., Control of vertebrate multiciliogenesis by miR-449 through direct repression of the Delta/Notch pathway. Nat Cell Biol, 2011. 13(6): p. 693-9.

27. Mori, M., et al., Notch3-Jagged signaling controls the pool of undifferentiated airway progenitors. Development, 2015. 142(2): p. 258-67.

28. Morimoto, M., et al., Canonical Notch signaling in the developing lung is required for determination of arterial smooth muscle cells and selection of Clara versus ciliated cell fate. J Cell Sci, 2010. 123(Pt 2): p. 213-24. 
29. Morimoto, M., et al., Different assemblies of Notch receptors coordinate the distribution of the major bronchial Clara, ciliated and neuroendocrine cells. Development, 2012. 139(23): p. 4365-73.

30. Pardo-Saganta, A., et al., Ciliated cells of pseudostratified airway epithelium do not become mucous cells after ovalbumin challenge. Am J Respir Cell Mol Biol, 2013. 48(3): p. 364-73.

31. Pardo-Saganta, A., et al., Parent stem cells can serve as niches for their daughter cells. Nature, 2015. 523(7562): p. 597-601.

32. Paul, M.K., et al., Dynamic changes in intracellular ROS levels regulate airway basal stem cell homeostasis through Nrf2-dependent Notch signaling. Cell Stem Cell, 2014. 15(2): p. 199-214.

33. Reid, A.T., et al., Blocking Notch3 Signaling Abolishes MUC5AC Production in Airway Epithelial Cells from Individuals with Asthma. Am J Respir Cell Mol Biol, 2020. 62(4): p. 513-523.

34. Rock, J.R., et al., Notch-dependent differentiation of adult airway basal stem cells. Cell Stem Cell, 2011. 8(6): p. 639-48.

35. Stupnikov, M.R., et al., Jagged and Delta-like ligands control distinct events during airway progenitor cell differentiation. Elife, 2019. 8.

36. Tata, P.R., et al., Dedifferentiation of committed epithelial cells into stem cells in vivo. Nature, 2013. 503(7475): p. 218-23.

37. Tata, P.R., et al., Airway-specific inducible transgene expression using aerosolized doxycycline. Am J Respir Cell Mol Biol, 2013. 49(6): p. 1048-56.

38. Tsao, P.N., et al., Epithelial Notch signaling regulates lung alveolar morphogenesis and airway epithelial integrity. Proc Natl Acad Sci U S A, 2016. 113(29): p. 8242-7.

39. Tsao, P.N., et al., Notch signaling controls the balance of ciliated and secretory cell fates in developing airways. Development, 2009. 136(13): p. 2297-307.

40. Tsao, P.N., et al., Notch signaling prevents mucous metaplasia in mouse conducting airways during postnatal development. Development, 2011. 138(16): p. 3533-43.

41. Xing, Y., et al., NOTCH1 is required for regeneration of Clara cells during repair of airway injury. Stem Cells, 2012. 30(5): p. 946-55.

42. $\mathrm{Xu}, \mathrm{K} ., \mathrm{N}$. Moghal, and S.E. Egan, Notch signaling in lung development and disease. Adv Exp Med Biol, 2012. 727: p. 89-98.

43. Zhang, S., et al., Jagged1 is the major regulator of Notch-dependent cell fate in proximal airways. Dev Dyn, 2013. 242(6): p. 678-86. 
44. Zuo, W., et al., p63(+)Krt5(+) distal airway stem cells are essential for lung regeneration. Nature, 2015. 517(7536): p. 616-20.

45. Kovall, R.A., et al., The Canonical Notch Signaling Pathway: Structural and Biochemical Insights into Shape, Sugar, and Force. Dev Cell, 2017. 41(3): p. 228-241.

46. Siebel, C. and U. Lendahl, Notch Signaling in Development, Tissue Homeostasis, and Disease. Physiol Rev, 2017. 97(4): p. 1235-1294.

47. Hendrix, A.Y. and F. Kheradmand, The Role of Matrix Metalloproteinases in Development, Repair, and Destruction of the Lungs. Prog Mol Biol Transl Sci, 2017. 148: p. 1-29.

48. Teoh, C.M., S.S. Tan, and T. Tran, Integrins as Therapeutic Targets for Respiratory Diseases. Curr Mol Med, 2015. 15(8): p. 714-34.

49. Wang, Z., et al., Insulin-Like Growth Factor-1 Signaling in Lung Development and Inflammatory Lung Diseases. Biomed Res Int, 2018. 2018: p. 6057589.

50. Baarsma, H.A. and M. Konigshoff, 'WNT-er is coming': WNT signalling in chronic lung diseases. Thorax, 2017. 72(8): p. 746-759.

51. Tsuchida, T. and S.L. Friedman, Mechanisms of hepatic stellate cell activation. Nat Rev Gastroenterol Hepatol, 2017. 14(7): p. 397-411.

52. Zhou, Y., et al., Extracellular matrix in lung development, homeostasis and disease. Matrix Biol, 2018. 73: p. 77-104.

53. Tschumperlin, D.J., Matrix, mesenchyme, and mechanotransduction. Ann Am Thorac Soc, 2015. 12 Suppl 1: p. S24-9.

54. Burgess, J.K., et al., The extracellular matrix - the under-recognized element in lung disease? J Pathol, 2016. 240(4): p. 397-409.

55. Vera, L., et al., Notch3 Deficiency Attenuates Pulmonary Fibrosis and Impedes Lung Function Decline. Am J Respir Cell Mol Biol, 2021.

56. McCauley, K.B., et al., Efficient Derivation of Functional Human Airway Epithelium from Pluripotent Stem Cells via Temporal Regulation of Wnt Signaling. Cell Stem Cell, 2017. 20(6): p. 844-857 e6.

57. $\mathrm{Hu}, \mathrm{Y}$., et al., Wnt/beta-catenin signaling is critical for regenerative potential of distal lung epithelial progenitor cells in homeostasis and emphysema. Stem Cells, 2020. 38(11): p. 1467-1478.

58. Hirano, K. and M. Namihira, LSD1 Mediates Neuronal Differentiation of Human Fetal Neural Stem Cells by Controlling the Expression of a Novel Target Gene, HEYL. Stem Cells, 2016. 34(7): p. 1872-82. 
59. Han, L., et al., The Notch pathway inhibits TGFbeta signaling in breast cancer through HEYL-mediated crosstalk. Cancer Res, 2014. 74(22): p. 6509-18.

60. Kuo, K.K., et al., Epigenetic inactivation of transforming growth factor-beta1 target gene HEYL, a novel tumor suppressor, is involved in the P53-induced apoptotic pathway in hepatocellular carcinoma. Hepatol Res, 2015. 45(7): p. 782-93.

61. Lavery, D.N., et al., Repression of androgen receptor activity by HEYL, a third member of the Hairy/Enhancer-of-split-related family of Notch effectors. J Biol Chem, 2011. 286(20): p. 17796-808.

62. Jalali, A., et al., HeyL promotes neuronal differentiation of neural progenitor cells. $J$ Neurosci Res, 2011. 89(3): p. 299-309.

63. Rea, M., et al., Genome-wide DNA methylation reprogramming in response to inorganic arsenic links inhibition of CTCF binding, DNMT expression and cellular transformation. Sci Rep, 2017. 7: p. 41474. 
A. Experimental design

- Lenti-Control or

Lenti-NICD3

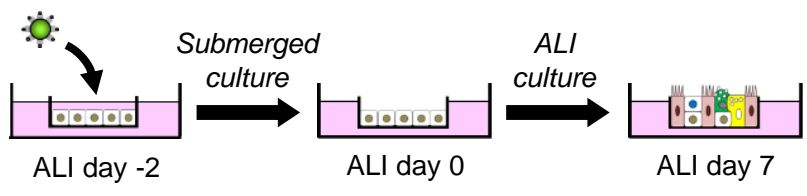

- Harvest cells at day 0, 3, 5 and 7 for bulk RNA-seq analysis

\section{B. GFP positivity}
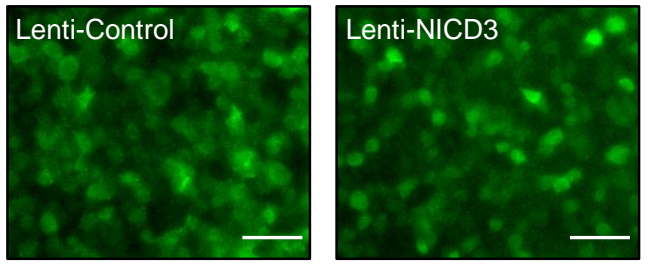

$\mathrm{Bar}=100 \mu \mathrm{m}$

\section{SCGB1A1 mRNA expression}

$\longrightarrow$ Lenti-Control ---O--- Lenti-NICD3
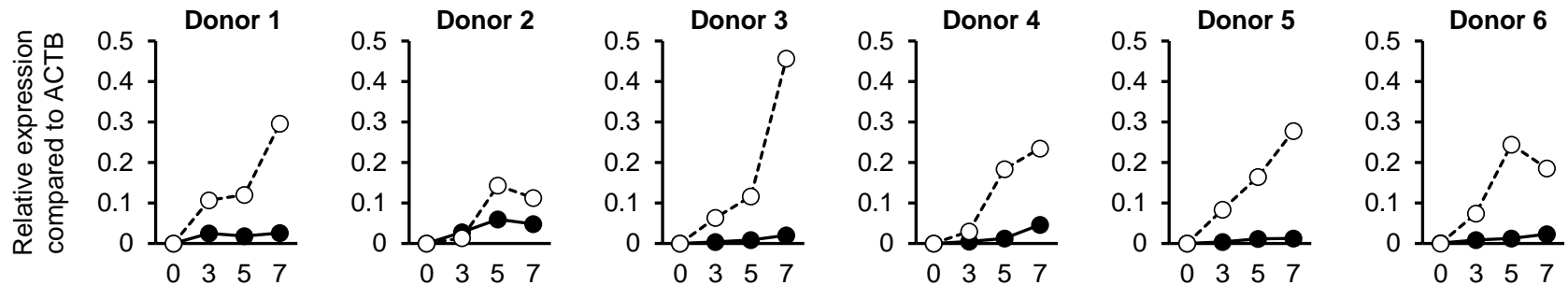

Days in ALI culture

\section{Top 10 pathways}

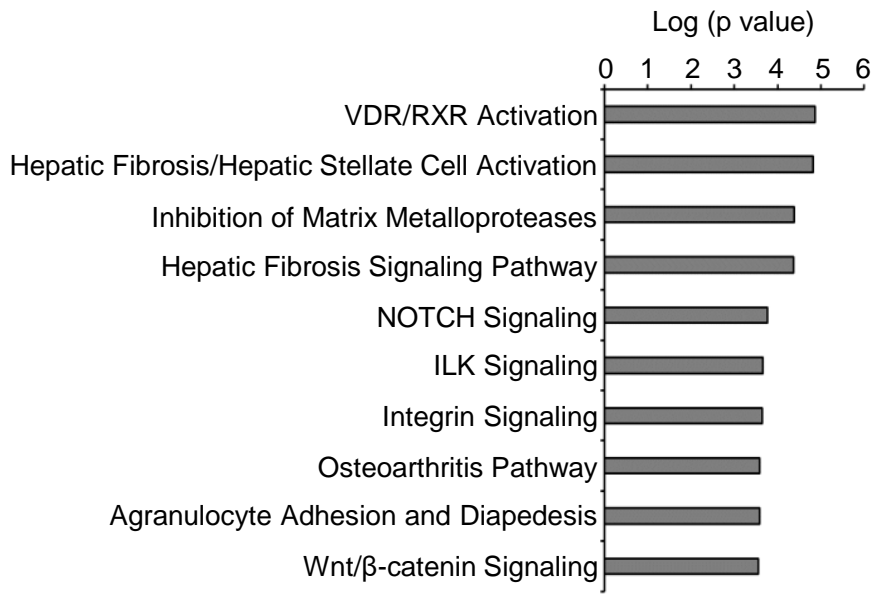

\section{F. NOTCH3 siRNA (ALI day 7)}

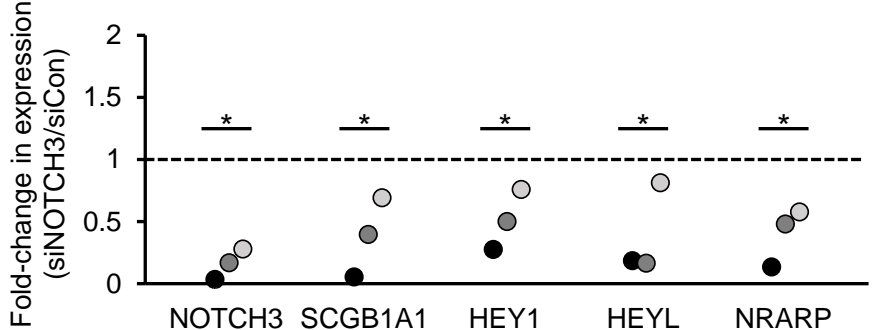

\section{E. NICD3 responsive genes}

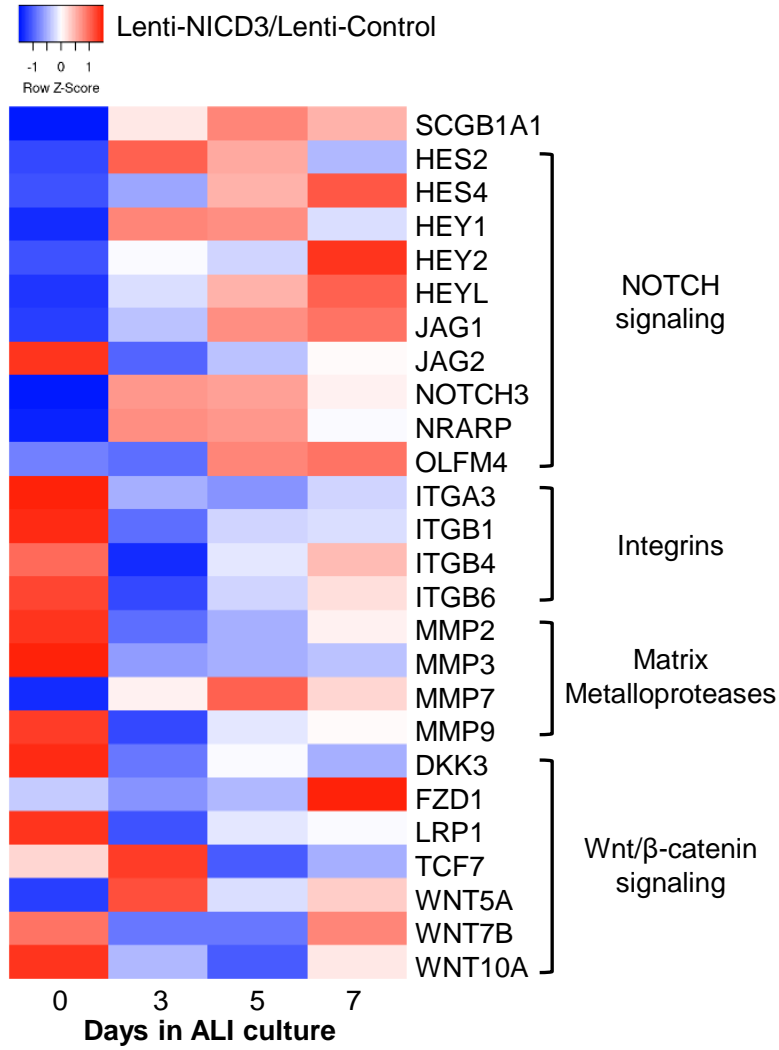


A. ALI day 0 vs. 7

Figure 2
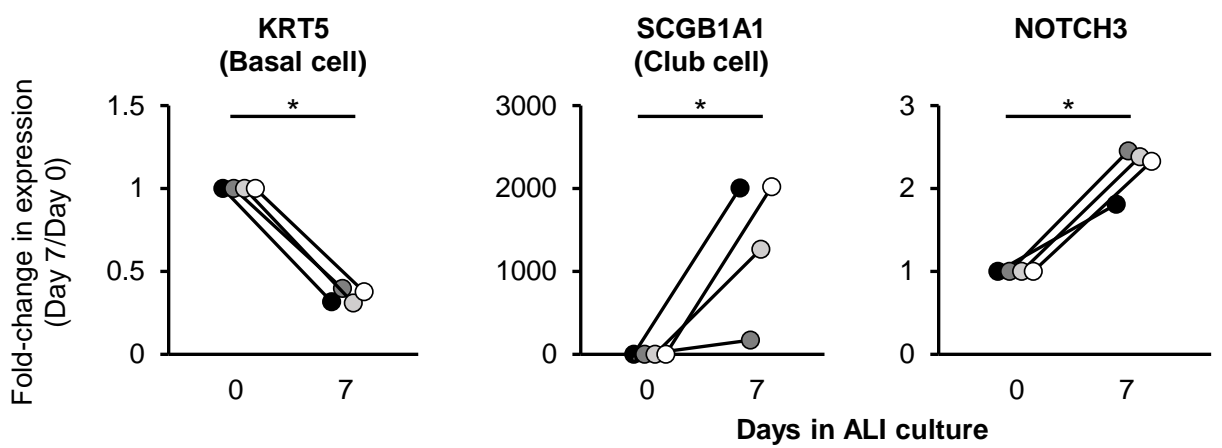

HEYL

\section{B. UMAP clustering}

Cluster 2

(4019 cells)

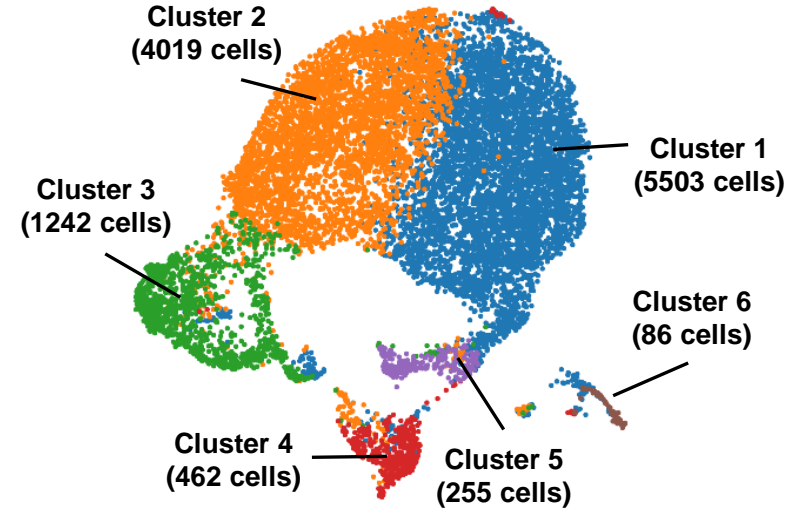

C. KRT5 expression

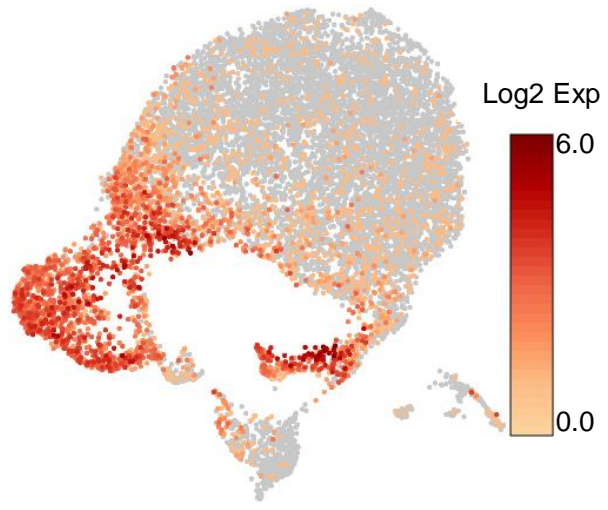

D. SCGB1A1 expression

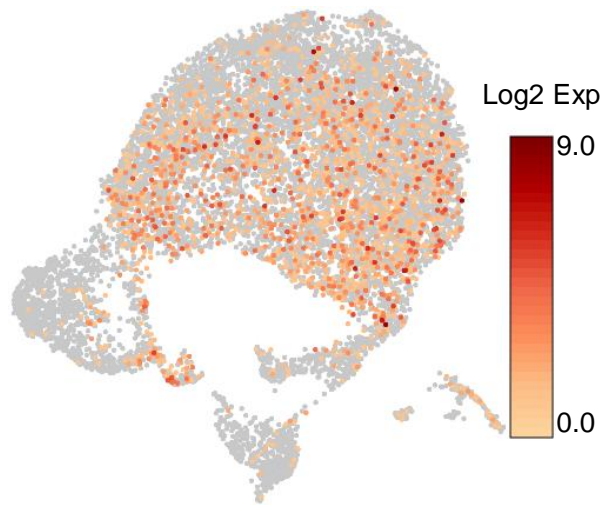

\section{E. NOTCH3 expression}
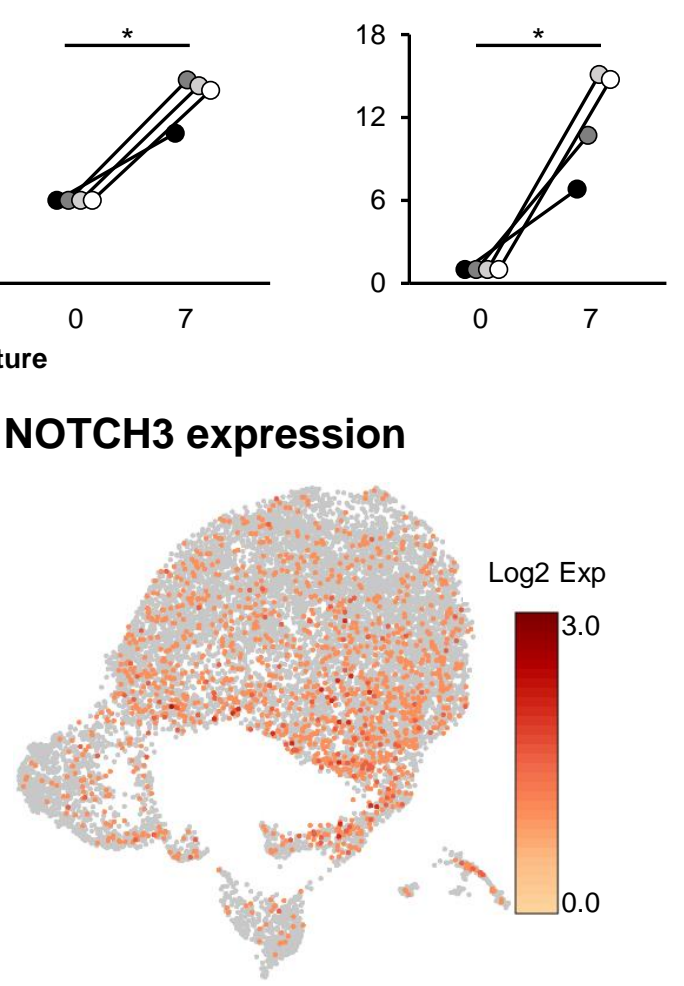

F. HEYL expression

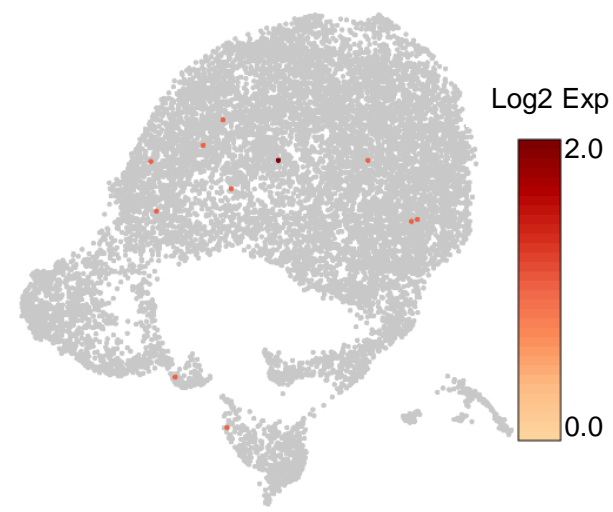

G. HEYL and SCGB1A1

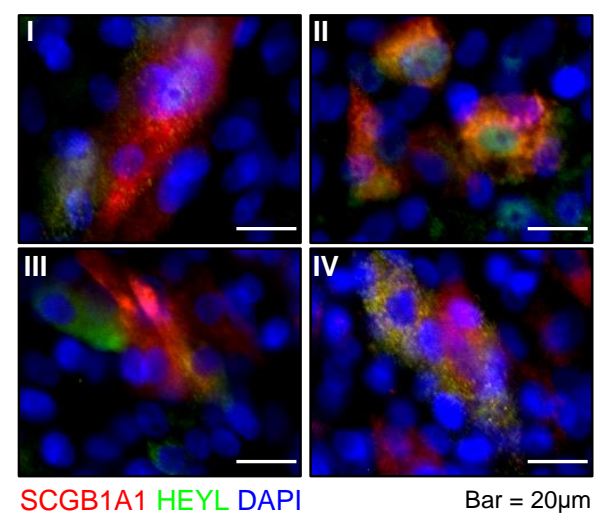


Figure 3

\section{A. Human}
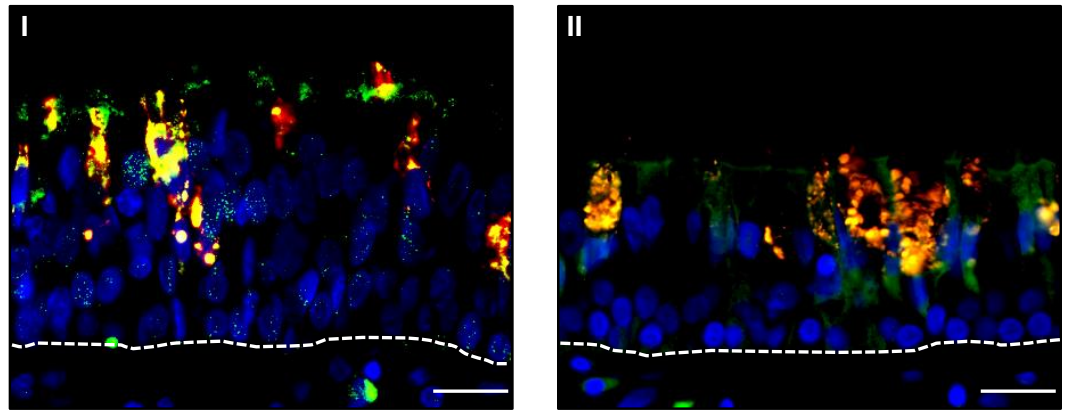

SCGB1A1 HEYL DAPI

\section{B. Mouse}
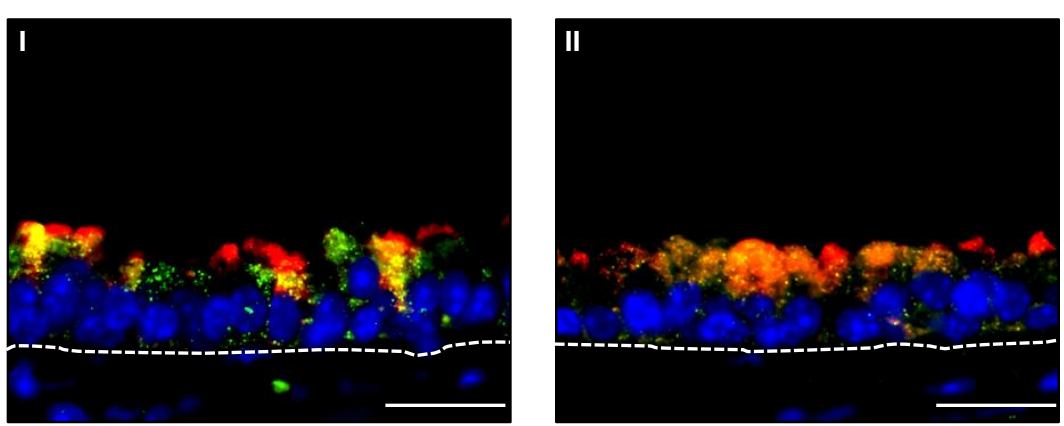

SCGB1A1 HEYL DAPI
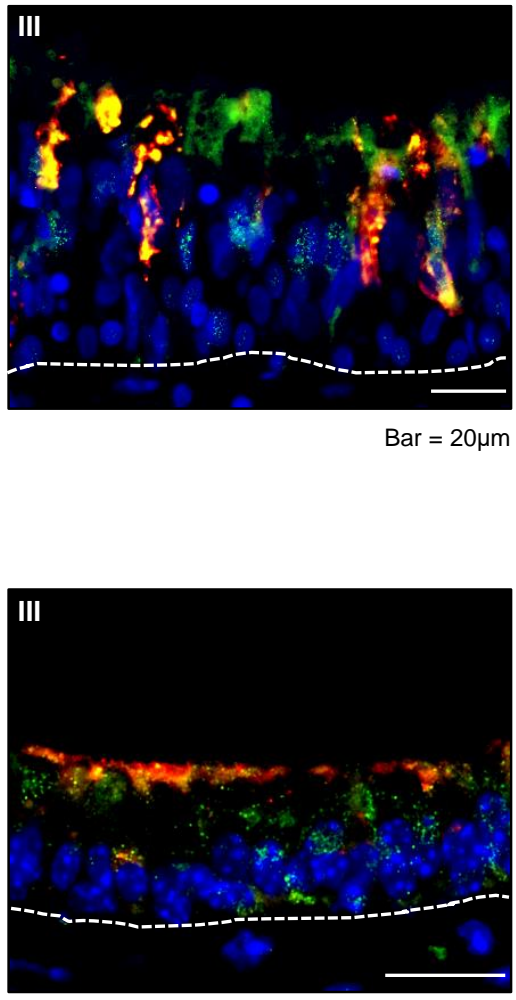

Bar $=20 \mu \mathrm{m}$ 
A. HEYL mRNA

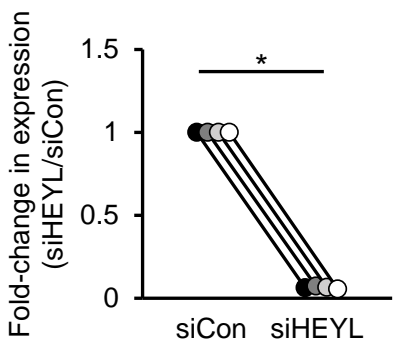

C. TEER

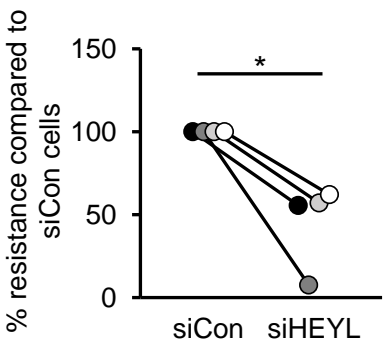

B. Morphology

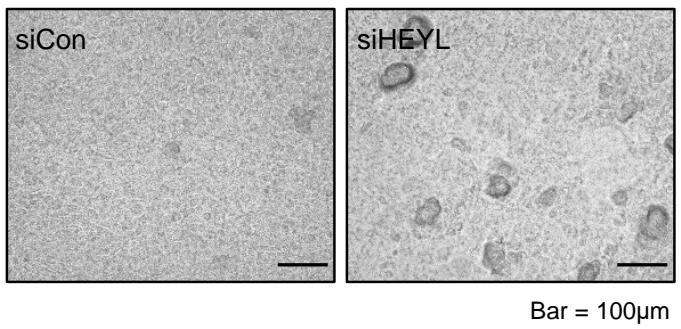

D. Tight junction genes, mRNA

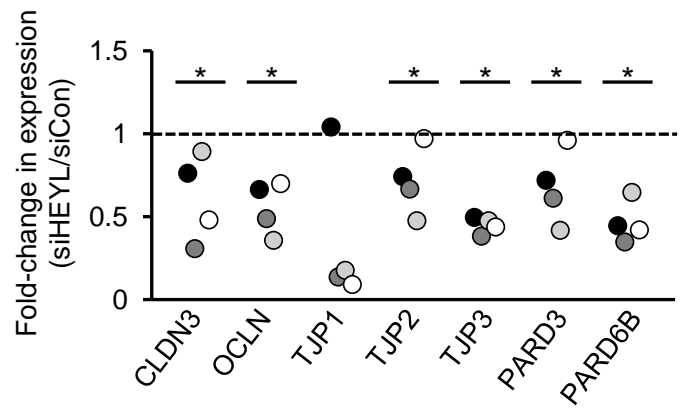

\section{E. SCGB1A1}

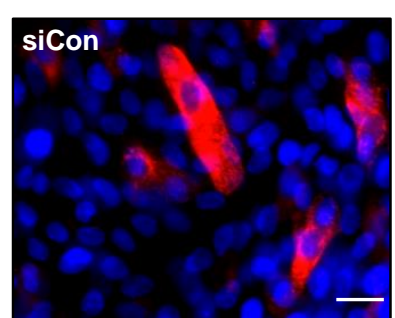

SCGB1A1 DAPI

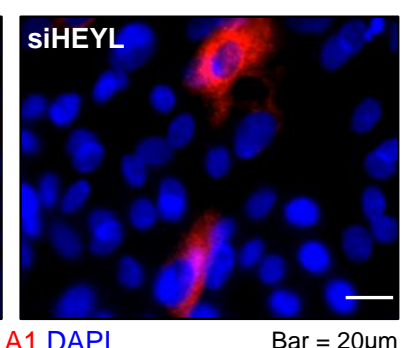

Bar $=20 \mu \mathrm{m}$

\section{F. KRT5}

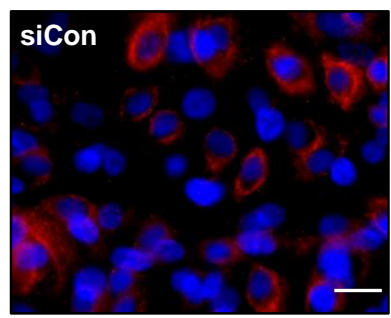

KRT5 DAP

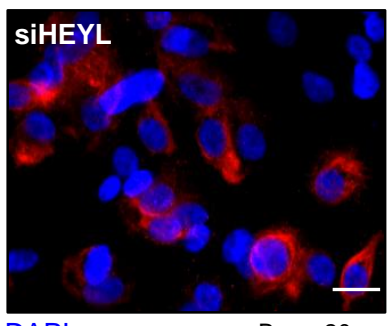

Bar $=20 \mu \mathrm{m}$

\section{G. KRT8}
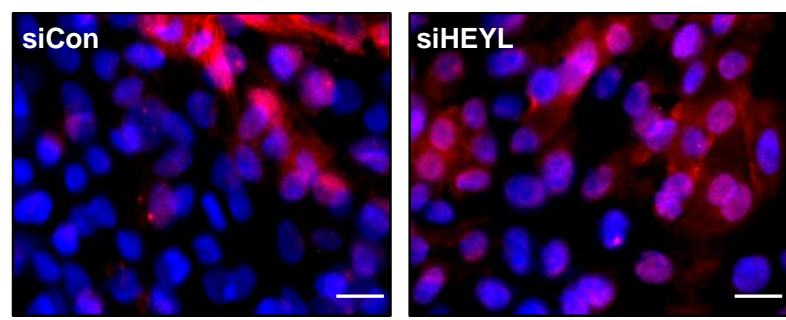

Bar $=20 \mu \mathrm{m}$
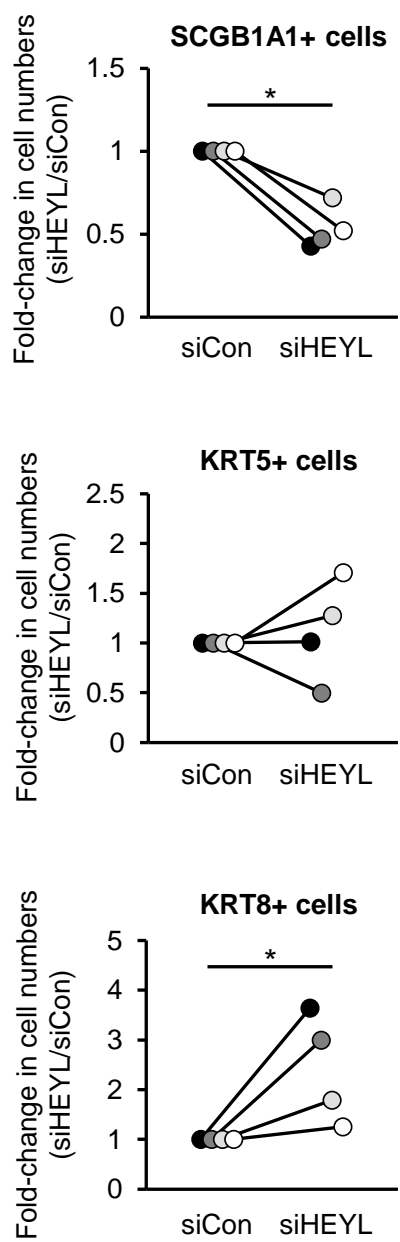